\title{
Les appareils générateurs de houle en laboratoire
}

\section{Laboratory wave generating apparatus}

\section{ÉTUDE THĚORIQUE D'UN CERTAIN TYPE D'APPAREIL A HOULE THEORETICAL STUDY OF A CERTAIN TYPE OF WAVE MACHINE}

\author{
PAR F. BIESEL ET F. SUQUET \\ F' UN GROUPE D'INGÉNIEURS AU LABORATOIRE DAUPHINOIS D'HYDRAULYUE (NEYRPIC, GRENOBLE)
} (Suite)

(Cf. la Houlle Blanche, $\mathrm{n}^{\circ} 2,1951$, page 147 et $\mathrm{n}^{\circ} 4$, 1951, page 475)

English synopsis, p. 699.

\section{Rappel du Sommaire}

\section{I. - INTRODUCTION}

$1^{0}$ Définition du problème étudié et notations.

2" Théories antérieures.

$$
\text { II. - DÉTAIL DU CALCUL. }
$$

$1^{\circ}$ Elude du problème homogène.

$2^{\circ}$ Recherche d'une solution particulière.

$3^{\circ}$ Détermination de la solution définitive.

III. - DISCUSSION DES RÉSULTATS

$1^{\circ}$ Indications générales.

$2^{\circ}$ Etude de quelques cas particuliers.

A. - Batteur piston :

a) Batteur piston émettant des houles longues.

b) Batteur piston émettant des houles courtes.

B. - Volet battant :

a) Volet battant émettant des houles longues.

b) Volet battant emettant des houles courtes.

\section{IV. - CONCLUSIONS GENERALES}

Notes ET COMPLEMENTS MATHEMATRQUES

1" Existence de la solulion du problème de décomposition posé par l'équalion 16.

$2^{\circ}$ Etude particulière des coefficients $c_{n}$ :

3" Justification des démonstrations formelles données dans le lexte de la note.

4. Etude des points singuliers.

$5^{\circ}$ Justification des conditions aux limites.

(Les deux premières parties ont été publiées dans le $n^{\circ} 2$ 1951.)

(Les $3^{\text {e }}$ et $4^{\mathrm{e}}$ parties ont été publiées rans le $n^{\circ} 4,1951$.) sent numéro.) 


\section{ANNEXES : NOTES ET COMPLEMENTS MATHEMATIQUES}

Dans cette annexe nous nous occuperons tout spécialement d'éclaircir les difficultés mathématiques introduites par l'emploi d'une série de fonctions. Par conséquent nous nous limiterons en principe à l'étude de la fonction :

$$
\Phi^{\prime}=\sum_{n=0}^{\infty} c_{n} o_{n} \cos k t
$$

la fonction $\Phi^{\prime \prime}$ ne présentant aucune difficulté. De même, nous étudierons essentiellement les termes d'ordre élevé de la série $\Sigma c_{n} \varphi_{n}$ et, mème quand nous ne le préciserons pas, il restera entendu que les formules et les énoncés ne s'appliquent pas nécessairement aux premicrs termes.

1. Existence de la solution de probrima: DE DÉcomposition posté par l'́oudTION (16).

Cette décomposition n'est possible dans le cas général que si la suite des fonctions :

$\operatorname{ch} m_{0} y, \quad \cos m_{1} y, \quad \cos m_{2} y, \quad \cos m_{3} y, \ldots \ldots$ est complète sur l'intervalle $(0, h)$.

Cette propriété peut être établie très rapidement en remarquant que ces fonctions sont les fonctions propres relatives aux valeurs propres :

$$
\lambda_{0}=-a_{0}{ }^{2}, \quad \lambda_{1}=a_{1}{ }^{2}, \lambda_{2}=a_{2}{ }^{2}, \lambda_{3}=a_{3}{ }^{2},
$$

de l'equation différentielle :

$$
U^{\prime \prime}+\lambda U=0
$$

où $U$ est une fonction de $y$ définie dans l'intervalle $(0, h)$ et satisfaisant aux conditions aux limites :

$$
\begin{aligned}
\mathrm{U}^{\prime}(0) & =0 . \\
\frac{l^{\prime \prime}}{g} \mathrm{U}(h) & =\mathrm{U}^{\prime}(h)
\end{aligned}
$$

Il résulte alors d'un théorème général que ces fonctions forment une suite non seulement complète, mais aussi orthogonale ${ }^{(1)}$.

Le fait que ces fonctions sont orthogonales permet de justifier instantanément les formules (18) et (19) qui peuvent s'écrire :

$$
c_{01}=\frac{\int_{0}^{h} \xi(x) \operatorname{ch} m_{0} x \cdot d x}{\int_{0}^{h} \operatorname{ch}^{2} m_{0} x \cdot d x}
$$

(1) A l'existence d'une valeur propre négative pres, il s'agit d'un cas particulier du probleme de StunLrovirle. On peut se référer par exemple à l'ouvrage : probleme der mathematischen Physili, de Covnast et Hilbert, $2^{\circ}$ edition, page 311.

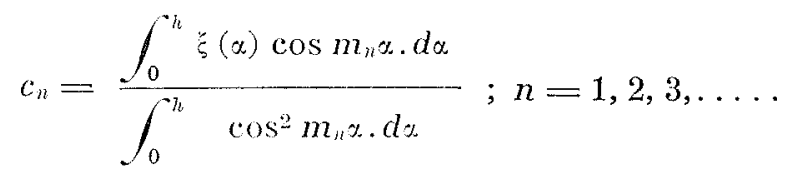

Il est à noter d'ailleur's que $\xi(y)$ ne satisfait pas en général aux conditions aux limites imposées à $U(y)$. En conséquence, il est possible que cette fonction ne soit approchée qu'en moyenne par la série (16). Cette restriction n'a pas de conséquence grave au point de vue physique. Il sera néanmoins bon de s'assurer que la fonction de courant $\Phi^{\prime}$ ne présente pas de singularité inadmissible aux extrémités du batteur. Nous reviendrons sur ce point plus loin.

\section{2" Etude phticulièe des confriciness $c_{n}$}

Nous devrons tout d'abord établir quelques propriétés des cocficients $m_{n}$ pour les grandes valeurs de $n$.

Nous savons que les coefficients $m_{n}$ sont les solutions de l'équation en $\alpha$ :

$$
k^{2}=\ldots-\alpha g \operatorname{tg} x h
$$

Nous savons également que l'on a :

$$
(n-1) \frac{\pi}{h}<m_{n}<n \frac{\pi}{h}
$$

Nous allons essayer de préciser cette inégalité pour les grandes valeurs de $n$. On peut écrire que $m_{n}$ satisfait à l'écquation (14) de la façon suivante :

$$
\operatorname{tg} m_{n} h=-\frac{j^{2} h}{g} \frac{1}{m_{n} h}
$$

Les équations (37) et (38) nous permettent ensuite d'écrire sans ambiguité :

$$
m_{n} h=n \pi-\operatorname{Arctg} \frac{k^{2} h}{g} \frac{1}{m_{n} h}
$$

Si nous nous limitons à considérer les parties principales, pour $n$ tendant vers l'infini, de toutes les grandeurs entrant dans le calcul on voit aisement que l'on a :

$$
\begin{aligned}
m_{n} h & \sim n \pi \\
\cos m_{n} h & \sim(-1)^{n} \\
\sin m_{n} h & \sim-(-1)^{n} \frac{k \cdot 2 h}{g} \frac{1}{n \pi}
\end{aligned}
$$

Nous allons maintenont pouroir aborder l'étude des coefficients $c_{n}$. On obtient par deux intégrations par parties sucessives la relation suivante: 


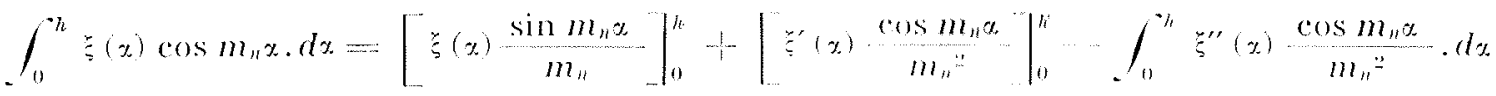

Cette relation s'écrit encore, compte tenu de (38):

$$
\left.\int_{0}^{h} \xi(x) \cos m_{n} x \cdot d x=\frac{1}{m_{n}^{2} !} ; \cos m_{n} h\left[\xi^{\prime}(h)-\frac{k^{2}}{g} \xi(h)\right]-\xi^{\prime}(0)-\int_{0}^{h} \xi^{\prime \prime}(x) \cos m_{n} x \cdot d x\right\}
$$

Ia derniere hypothèse que nous avons formulée sur la fonction $\xi(y)$, à savoir que la dérivée troisième $\xi^{\prime \prime \prime}(y)$ était bornée sauf en un nom- bre fini de points singuliers que nous désignerons ci-après par $y_{1}, y_{2}, y_{3}, \ldots$ ete. dans l'ordre croissant, nous permet d'écrire :

$$
\int_{0}^{h} \xi^{\prime \prime}(x) \cos m_{n} x \cdot d x=\left[\xi^{\prime \prime}(x) \frac{\sin m_{n} y}{m_{n}}\right]_{0}^{y_{1} \cdots \cdot 0}+\left[\xi^{\prime \prime}(x) \frac{\sin m_{n} y}{m_{n}}\right]_{y_{1}+0}^{y_{n}-0}+\ldots-\int_{n}^{\prime \prime} \xi^{\prime \prime \prime} \frac{\sin m_{n \prime}^{\prime \prime}}{m_{n}} \cdot d x
$$

Ce développement nous montre que l'intégrale du premier membre tend vers zéro quand $n$ lend vers l'infini. En nous limilant à nouveau aux parties principales, nous pouvons donc écrire :

$$
\int_{0}^{h} \xi(x) \cos m_{1} z \cdot d z \sim(-1) n\left[\xi^{\prime}(h)-\frac{k^{2}}{g} \zeta(h)\right] h^{2} \cdot-\frac{\xi^{\prime}(0) h^{2}}{\pi^{2} n^{2}}
$$

I'où l'on tire :

$$
c_{n} \sim \frac{2 h}{\pi^{2}} !(-1)^{n}\left\lceil\check{\zeta}^{\prime}(h)-\frac{l^{2}}{g} \xi(h) \mid \cdots \cdots(0) \frac{1}{n^{2}}\right.
$$

\section{3" JUSTHFATION DMS DEMONSTRATONS FORMELLES DONNÉS DANS LE THXTE DE LA NOTE}

Dans le texte de la note nous avons reproduit les démonstrations formelles de M. HAverock en admettant en particulier sans examen que la série $\varphi^{\prime}=\Sigma c_{n} \varphi_{n}$ avait une somme et que l'on avait le droit de la dériver terme à terme pour montrer que ọ́ satisfaisait à toutes les conditions énoncées. On peut justifier cette manière de faire pour le cas particulier que nous étudions.

Afin de rendre ces démonstrations rigoureuses, nous remarquerons tout d'abord que les fonetions ôn sont les parties entières des fonctions de la variable imaginaire $z=x+i y$ :

$$
Z_{n}(z)=o_{n}+i \omega_{n}=\frac{k}{m_{n}} e^{-m_{n} z}
$$

dans le domaine étudie la fonction $Z_{n}$ est holomorphe et l'on a :

$$
\left|Z_{n}\right| \leqslant \frac{k}{m_{n}}
$$

Les relations (46), (47) et (48) montrent donc sans ambiguité que les séries :

$$
\varphi^{\prime}=\mathrm{\Sigma} c_{n} \vartheta_{n} \text { et } Z^{\prime}=\mathrm{\Sigma} c_{n} \mathrm{Z}_{n}
$$

sont uniformément convergente dans le domaine étudié puisque le terme général de la série est au plus de la forme $\frac{A}{n^{3}}$, A étant borné.

In théorème général (WErrastruss) nous per- met done d'affirmer que la série $\mathrm{Z} c_{n} Z_{n}$ est dérivable terme à terme, c'est-à-dire que si la somme $\Sigma c_{n} \frac{\partial^{\mu} Z_{n}}{\partial z^{\mu}}$ converge, elle a pour limite $\frac{\partial p Z^{\prime}}{\partial z^{p}}$ quelque soit $p(1)$. On déduit immédiatement de ce thérème que la série $y_{n}$ on est également dérivable terme à terme (e'est-à-dire que) :

$$
\frac{\partial p^{n} q}{\partial x^{p} \partial y^{\prime}}=\operatorname{Lim}_{n \rightarrow \infty} \stackrel{n}{ \pm} c_{n} \frac{\partial p^{+} \varphi_{n}}{\partial x^{n} \partial y^{q}}
$$

lorsque celle limite existe, et par conséquent que nos manipulations mathématiques sont justifiées.

\section{4" ETUd: des ponts singulares}

Les résultats que nous venons d'obtenir nous permettent d'étudier aisément l'écoulement représente par la fonction :

$$
w^{\prime}=\rho^{\prime} \cos k t
$$

Les vitesses correspondantes seront données par :

$$
\begin{aligned}
& u^{\prime}=\frac{\partial y_{p}^{\prime}}{\partial u} \cos k t \\
& v^{\prime}=\frac{\partial \vartheta^{\prime}}{\partial y} \cos k t
\end{aligned}
$$

(1) Voir par exemple le Cours d'Analyse mathematique, de M. Goursat, 6\% édition, p. 667. 
Nous sommes donc conduits à étudier les dérivées $\frac{\partial \varphi^{\prime}}{\partial x}$ et $\frac{\partial \varphi^{\prime}}{\partial y}$. Nous venons de voir que l'on a :

$$
\begin{aligned}
& \frac{\partial \varphi^{\prime}}{\partial x}=\Sigma c_{n} \frac{\partial \varphi_{n}}{\partial x} \\
& \frac{\partial \varphi^{\prime}}{\partial y}=\Sigma c_{n} \frac{\partial \varphi_{n}}{\partial y}
\end{aligned}
$$

Si nous nous reportons aux expressions de $\varphi_{n}$, on voit que l'on a (pour $n>0$ ):

$$
\begin{aligned}
& \frac{\partial \varphi_{n}}{\partial x}=-k \cos m_{n} y e^{-m_{n} x} \\
& \partial g_{n}=-k \sin m_{n} y e^{-m_{n} x} \\
& \partial y
\end{aligned}
$$

Les valeurs absolues de $\frac{\partial o_{n}}{\partial x}$ et $\frac{\partial o_{n}}{\partial y}$ étant étant inférieures à $k$ dans le domaine étudié, et compte tenu de (46), nous voyons que les termes des séries (52) et (53) ont une valeur principale tout au plus en $\frac{\mathrm{A}}{n^{2}}$, A étant une constante. Nous sommes donc assurés que ces séries convergent et que $u$ et $v$ sont « finis » dans tout le plan. Il est à noter d'ailleurs qu'étant donné nos hypothèses sur la petitesse des mouvements, l'adjectif fini signifie en réalité du même ordre que les valeurs de $\xi(y)$, c'est-á-dire infiniment petit du premier ordre. Ces conclusions resteront valables pour les déplacements.

\section{$5^{\circ}$ JUSTIFICATION DES CONDITIONS AUX LIMITES}

Conformément à un théorème classique, nous avons exprimé les conditions aux limites sur les limites du liquide au repos et non sur ses limites vraies. Cette facon de faire n'est justifiée que si :

$1^{\circ}$ Les déplacements des particules sont des infiniment petits du premier ordre. (L'infiniment petit du premier ordre servant de base au calcul étant par exemple une valeur de $\zeta(y)$. Nous venons de voir que cette condition est réalisée.

$2^{\circ}$ Les vitesses sur la limite fictive ainsi choisic ne diffèrent des vitesses correspondantes sur la limite réelle que par des termes du second ordre.

Cette condition est automatiquement réalisée si les dérivées partielles de la vitesse :

$\frac{\partial u^{\prime}}{\partial x}, \frac{\partial u^{\prime}}{\partial y}, \frac{\partial v^{\prime}}{\partial x}, \frac{\partial v^{\prime}}{\partial y}$, sont partout «finies»,

c'est-à-dire sont des infiniment petits du mème ordre que les vitesses. Nous n'insistons pas sur ce cas classique qui est bien connu. Malheureusement dans le problème actuel nous ne sommes plus assurés que les dérivées partielles de la vi- tesse soient finies, au contraire, elles peuvent être localement infinies.

Examinons tout particulièrement ce point.

$\mathrm{Si}$ nous formons les dérivées de la vitesse, nous voyons immédiatement que l'on ne peut plus conclure automatiquement à la convergence des séries obtenues. En effet, leurs termes ont des parties principales en $\frac{A}{n}$. Un examen plus approfondi devient donc nécessaire. Etudions par exemple la dérivée $\frac{\partial u^{\prime}}{\partial x}$, il vient :

$$
\frac{\partial u^{\prime}}{\partial x}=\frac{\partial^{2} \varphi^{\prime}}{\partial x^{2}}=\Sigma c_{n} k m_{n} \cos m_{n} y e^{-m_{n} v}
$$

En remplaçant $c_{n}$ et $m_{n}$ par leurs valeurs principales, cette série devient :

$$
\begin{gathered}
\sum \frac{2 k}{\pi}\left\{(-1)^{n}\left[\xi^{\prime}(h)-\frac{k^{2}}{g} \xi(h)\right]\right. \\
\left.-\xi^{\prime}(0)\right\} \frac{1}{n} \cos m_{\mu} y e^{-m_{n^{k}}}
\end{gathered}
$$

Etudions par exemple cette série au point $x=0, y=0$. Nous voyons immédiatement qu'elle est divergente si $\xi^{\prime}(0)$ est différent de zéro.

Par conséquent $\frac{\partial u^{\prime}}{\partial x}$ est infini au pied du batteur si $\xi(y)$ ne satisfait pas à la condition aux limites.

$$
\xi^{*}(y)=0
$$

On verrait de même aisément qu'il y a une singularité du même genre au sommet du batteur si la condition aux limites correspondante :

$$
\xi^{\prime}(h)-\frac{k^{2}}{g} \xi(h)=0
$$

n'est pas satisfaite.

Il semble done que nous n'ayons pas le droit de confondre la vitesse horizontale de l'eau au voisinage du batteur avec la vitesse au voisinage de l'axe des $y$ puisque dans cette région le taux de variation de $u$ en fonction de $x$ peut devenir infini. Nous allons montrer que cette approximation reste pourtant valable.

Pour cela, nous allons étudier directement les valeurs de $u$ au voisinage des points où $\frac{\partial u}{\partial x}$ risque de devenir infini.

La série définissant $u$ peut s'écrire :

$$
\Sigma-c_{n} k \cos m_{n} y e^{-m_{n} x}
$$


En remplaçant $c_{n}$ par sa valeur principale, le terme général de celle série devient :

$$
-k \frac{2 h}{\pi^{2}}(-1)^{n}\left[\xi^{\prime}(h)-\frac{k^{2}}{g} \xi(h)\right]-\xi^{\prime}(0) \frac{1}{n^{2}} \cos m_{n} y e^{-m_{n} n^{2}}
$$

La valeur principale de ce terme est identique à celle de l'expression :

$$
-k \frac{2 h}{\pi^{2}}\left\{(-1)^{n}\left[\xi^{\prime}(h)-\frac{k^{2}}{g} \zeta(h)\right]-\xi^{\prime}(0) \frac{1}{n(n-1)} \cos n \pi \frac{y}{h} e^{-n \pi \alpha / h}\right.
$$

La nouvelle série ainsi obtenue représente, aux premiers termes près, le développement de la partie réelle de la fonction :

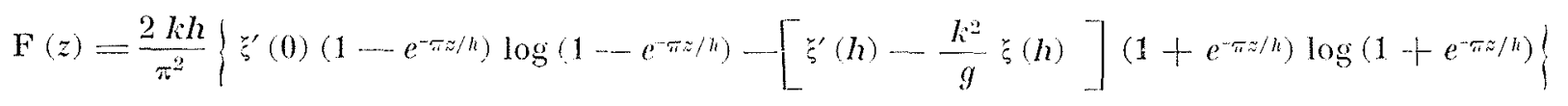

En d'autres termes, la vitesse $u$ se compose de la somme de termes finis et ayant une dérivée finie par rapport à $x$ et de la partie réelle de la fonction $(60)$.

Il nous suffira donc d'étudier la façon dont se comporte cette dernière fonction.

Etudions par exemple ce qui se passe au voisinage de l'origine où $\frac{\partial u}{\partial x}$ devient infini. Etudions la valeur de la vitesse au point $\Delta z$, étant entendu que $\Delta z$ est infiniment petit du premier ordre.

Remarquons qu'il suffira d'ctudier le terme en $\xi^{\prime}(0)$, l'autre terme étant régulier à l'origine. Il nous reste donc à étudier (au seeond ordre près) :

$$
\frac{2 k h}{\pi^{2}} \xi^{\prime}(0) \frac{\pi \Delta z}{h} \log \frac{\pi \Delta z}{h}
$$

Nous voyons done que si l'on désigne par $\varepsilon$ un infiniment petit du premier ordre, l'erreur faite sur $u$, en se placant à une distance de l'origine de l'ordre de grandeur d'e, n'est que de l'ordre de grandeur de :

$$
\varepsilon^{2} \log \varepsilon
$$

Cette quantité n'est pas rigoureusement un infiniment petit du second ordre, elle est cependant infiniment plus petite que toute quantité d'ordre un tant soit peu inférieur au second.

Nons n'avons fait cette démonstration que pour l'origine, mais on pourrait l'étendre sans difficulté aux autres points litigieux.

\section{CONCLUSION DE L'ANNEXE}

Les démonstrations effectuées dans cette annexe ont pour intérêt essentiel de montrer que, dans le cas d'un batteur dont la forme satisfait aux conditions énoncées, les équations obtenues par la méthode de Havelock ne comportent pas de singularité inadmissible sur le plan physique et que, par conséquent, les conclusions théoriques auxquelles elles conduisent donnent probablement une très bonne représentation de la réa- lité. Rappelons d'ailleurs que les restrictions imposées à la forme du batteur au moment de son élongation maximum ne sont pratiquement pas différentes de celles que le bon sens de l'hydraulicien aurait exigées, à savoir l'absence de décrochements brusques ou de points anguleux. La restriction sur les courbures, qui n'est d'ailleurs pas strictement indispensable, ne sera jamais gênante dans les cas réels. 


\section{Tableau synoptique}

\section{des différents appareils générateurs de houle}

La première partie de l'article de M. Biesel, publiée dans notre numéro 2/1951, était précédée de quelque pages de M. Suquet intitulées : «Caractéristiques et essai de classification des appareils générateurs de houle».

Dans cet avant-propos, l'auteur annonçait la publication, répartie tout au long de l'article de M. Brésel, de fiches donnant, pour chacun des types d'appareils envisagés, un certain nombre de renseignements sur sa construction, son pouvoir réfléchissant, sa mobilité, etc., ainsi que sur son comportement dans le cadre de la théorie de M. Breser.

Les fiches n"s 1 à 4 ont paru dans le numéro 2/1951; les fiches 5 à 11 dans le numéro 4/1951; le lecteur trouvera dans les pages qui suivent les fiches 12 a 16.

M. Suquer avait cru bon de présenter, en tête de ces fiches, un tableau synoptique; nous pensons faciliter la tâche de nos lecteurs en reproduisant ce tableau ci-dessous.

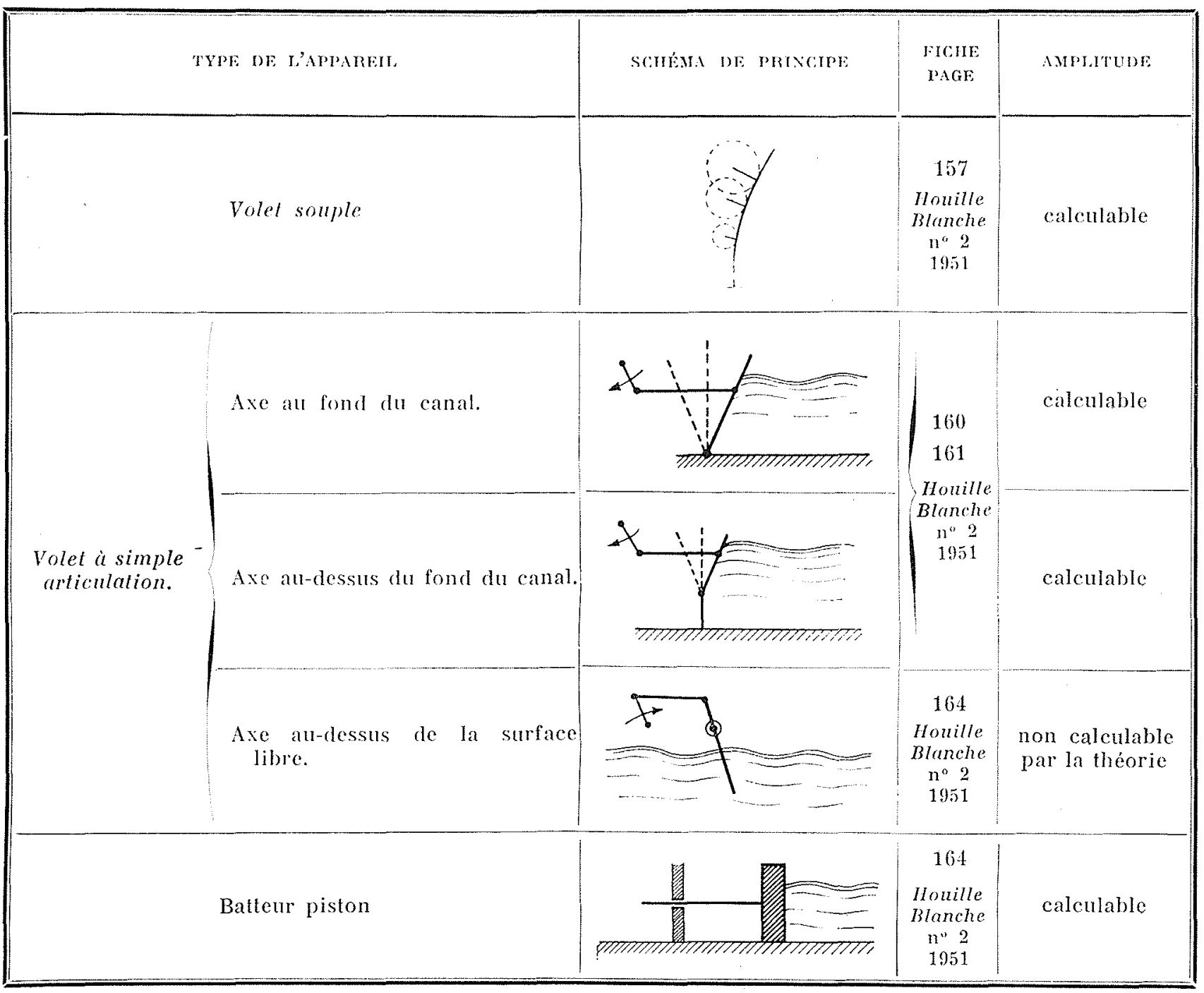




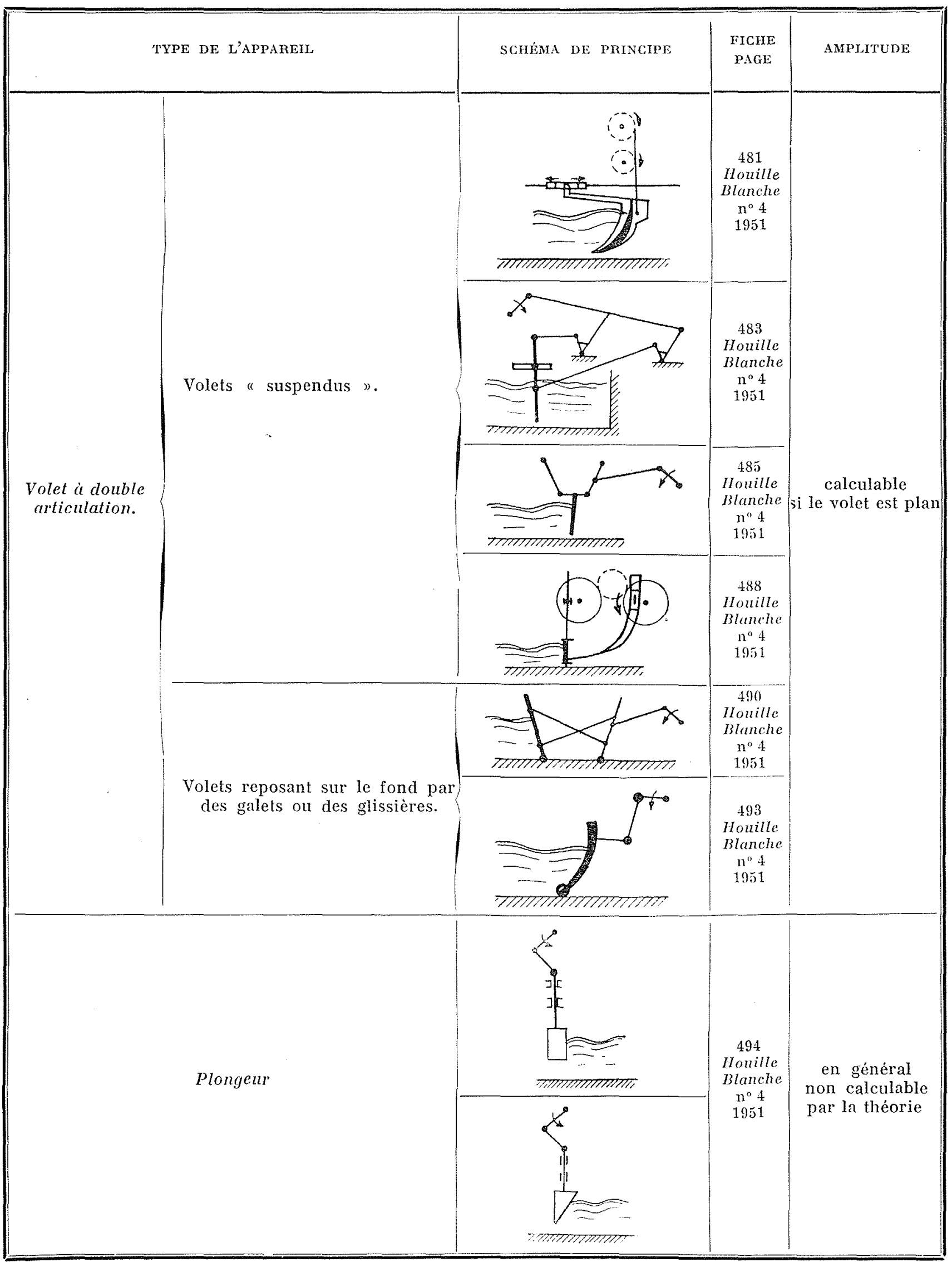




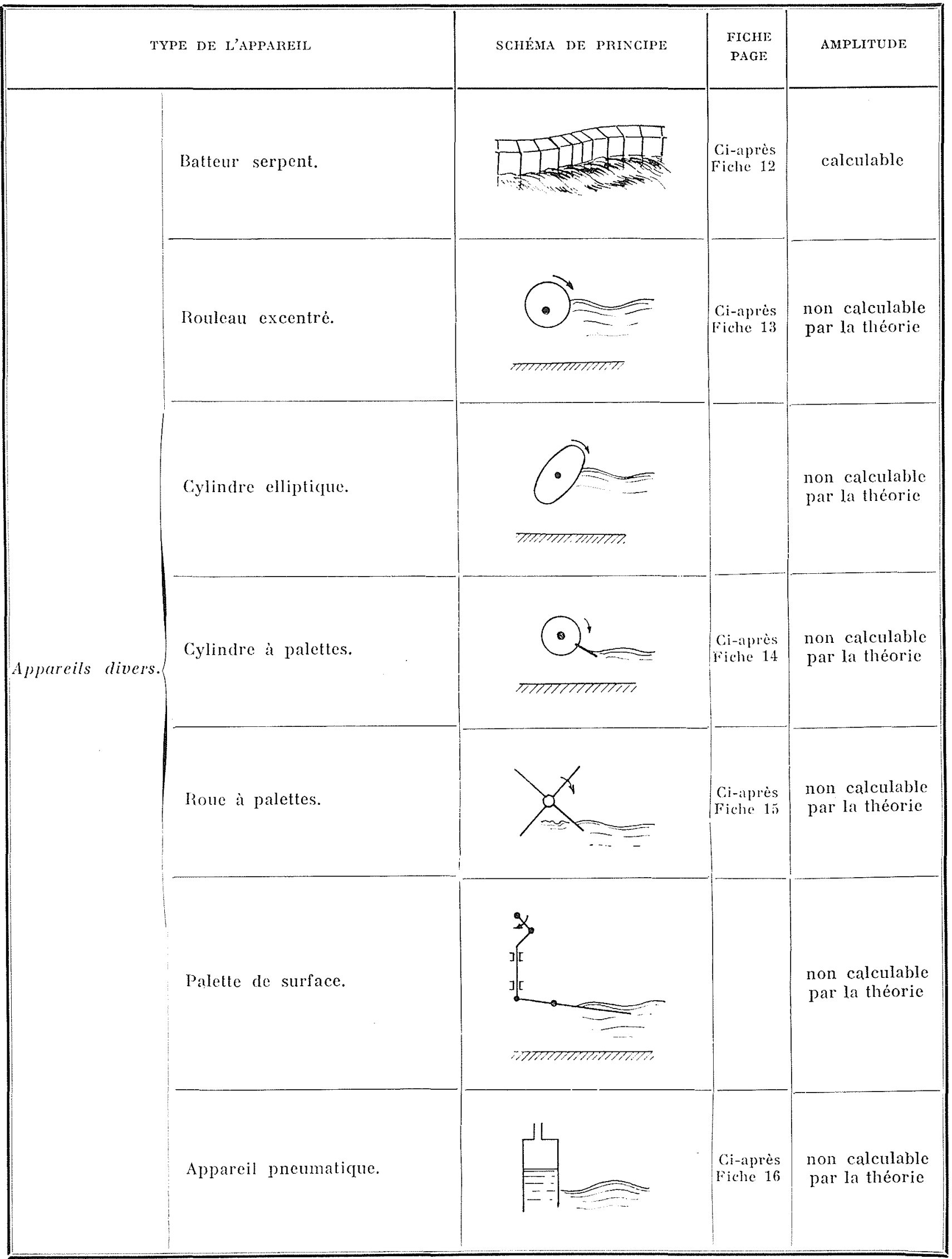




\section{FICHE N"12}

\section{Batteur serpent}


Element de balteur.

Batteur serpent.

\section{Principe :}

Ce batteur se compose d'une rangée de volets de même hauteur, de largeur faible et oscillant autour du même axe géométrique; l'aspect de ce batteur rappelle un clavier de piano.

Un mécanisme approprié - un arbre à cames par exemple - permet de donner a ces batteurs des mouvements d'oscillation autour de leur axe commun, de même période, mais régulièrement déphasés les uns par rapport aux autres. Chaque batteur considéré isolément émet une onde élémentaire sensiblement circulaire; l'ensemble de ces ondes admot it chaque instant une enveloppe qui constitue la cròte de la houle émise par le batteur.

On peut ainsi produire des loules dont la direetion des lignes de crête peut varier sans cu'on ait a changer l'orientation du batteur. Ceci peut ctre très précicux dans certains modèles' à trois dimensions.

\section{Construction :}

Elle est malheureusement assez onéreuse el délicate. 


\section{FICHE N*13}

\section{Rouleau excentré}

\section{Principe. - Loi du movenent :}

Cylindre circulaire tournant autour d'un axe parallèle à son axe géométrique et excentré par rapport à celui-ci.

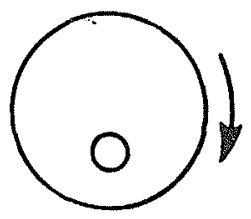

Roulenu excentré

Le mouvement imprimé aux particules d'eau au voisinage du rouleau est assez éloigné de celui de la houle, mais il se régularise à mesure que la distance parcourue augmente et l'on obtient finalemen: une houle acceptable si la profondeur est suffisamment grande, du moins pour les faibles cambrures.

\section{CALCUL DE L'AMPLITUDE DE LA HOULE PRODUTTE :}

La théorie exposée dans cet article ne peut evidemment donner la valeur de l'amplitude de la houle obtenue.

\section{Construction :}

Elle peut être très simple; on peut en eflet uliliser. un simple tuyau en tôle auquel sont adaptés deux flascues aux extrémités. On peut alléger la construction en utilisant pour le rouleau une carcasse en bois recouverte de tôle légère.

\section{INERTE :}

Du fait même de son principe, l'inertie de l'appareil est assez grande; la dernière solution indi- quée, évidemment plus coûteuse, permet de la diminuer. Ce dernier point est important; en efret, l'appareil doit souvent avoir une longueur importante s'il est employé sur un bassin a houle; par suite, si l'on u'ilise un tuyau métallique, la tôle qui le constitue doit avoir une épaisseur relativement grande pour assurer une bomne rigidité.

\section{ENTRETIEN :}

L'expérience montre que l'on obtient la houle de qualité optimum en immergeant prescue complètement le cylindre. Dans ces conditions, les paliers ainsi que le système de réglage de l'amplitude se trouvent dans l'eau; il peut en résulter quelques inconvénients.

\section{POUVOIR RÉFLÉChISSANT :}

La génératrice inférieure du rouleau dans sa position la plus basse étant assez éloignée du fond du bassin, le coefficient de réflexion est moins élcvè que dans le cas des volets.

\section{RÉglage :}

Le réglage de l'amplitude se fait en modifiant l'excentrement de l'axe de rotation du rouleau. L'expérience montre que sur les batteurs de grande dimension elle est longue et assez délicate.

\section{Monilíné :}

Le cylindre ainsi que son mécanisme peuvent ètre montés sur un bâti qui peut être orienté facilement dans la direction choisie. Le croquis ci-contre montre un tel ensemble destiné à un bassin à houle. 


\section{FICHE N*13}

(SUITE)
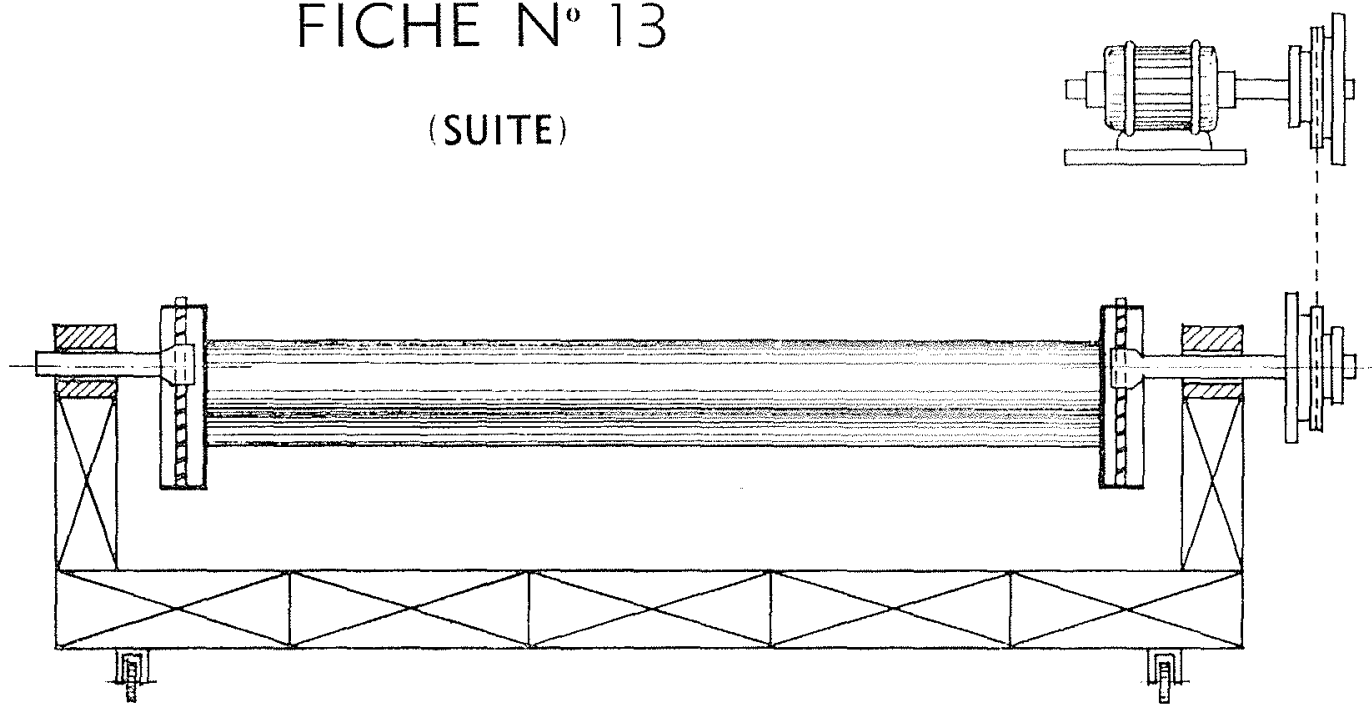

Croquis communiqué par le Laboraloire Dauphinois c'Hydranlique (Neyrpic).

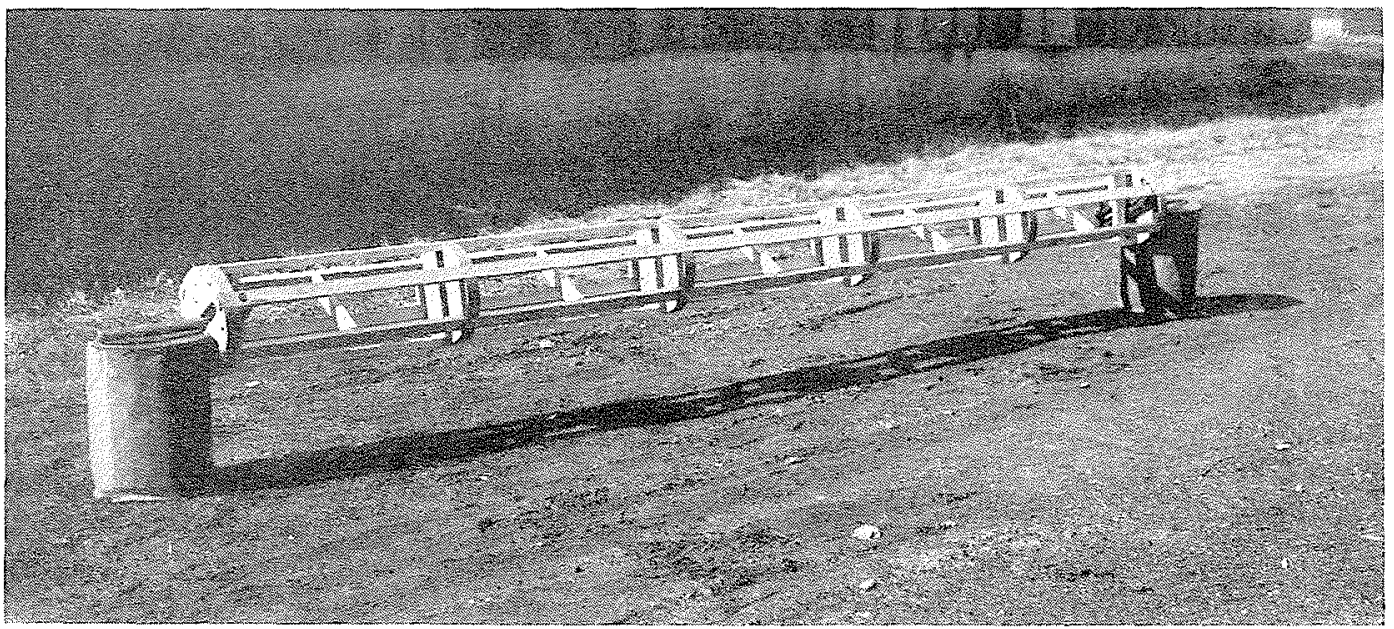

Photographie communique par le « Beach Erosion Bourd\%.

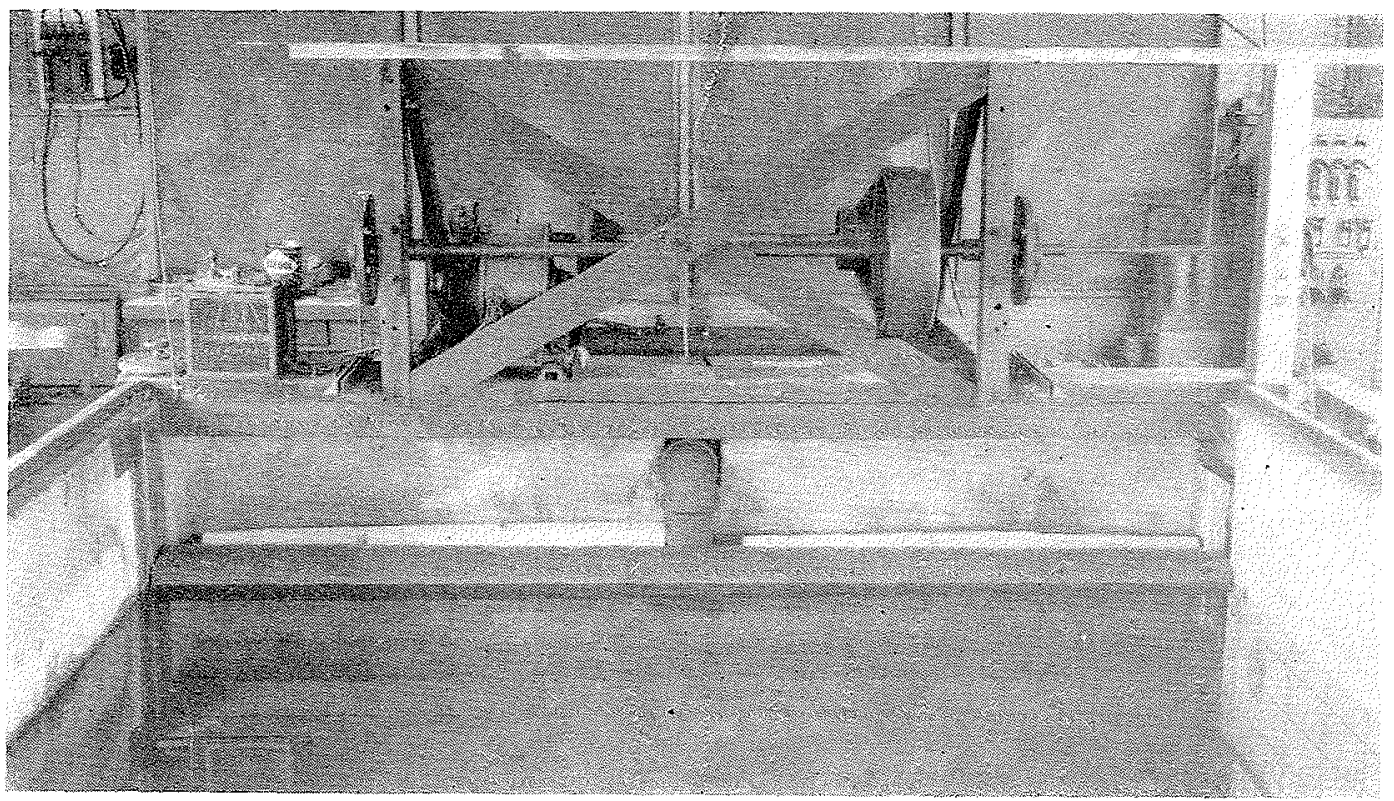

Photographie communiquée par le « Beach Frosion Board ». 


\section{FICHE No 14}

\section{Cylindre à palette}

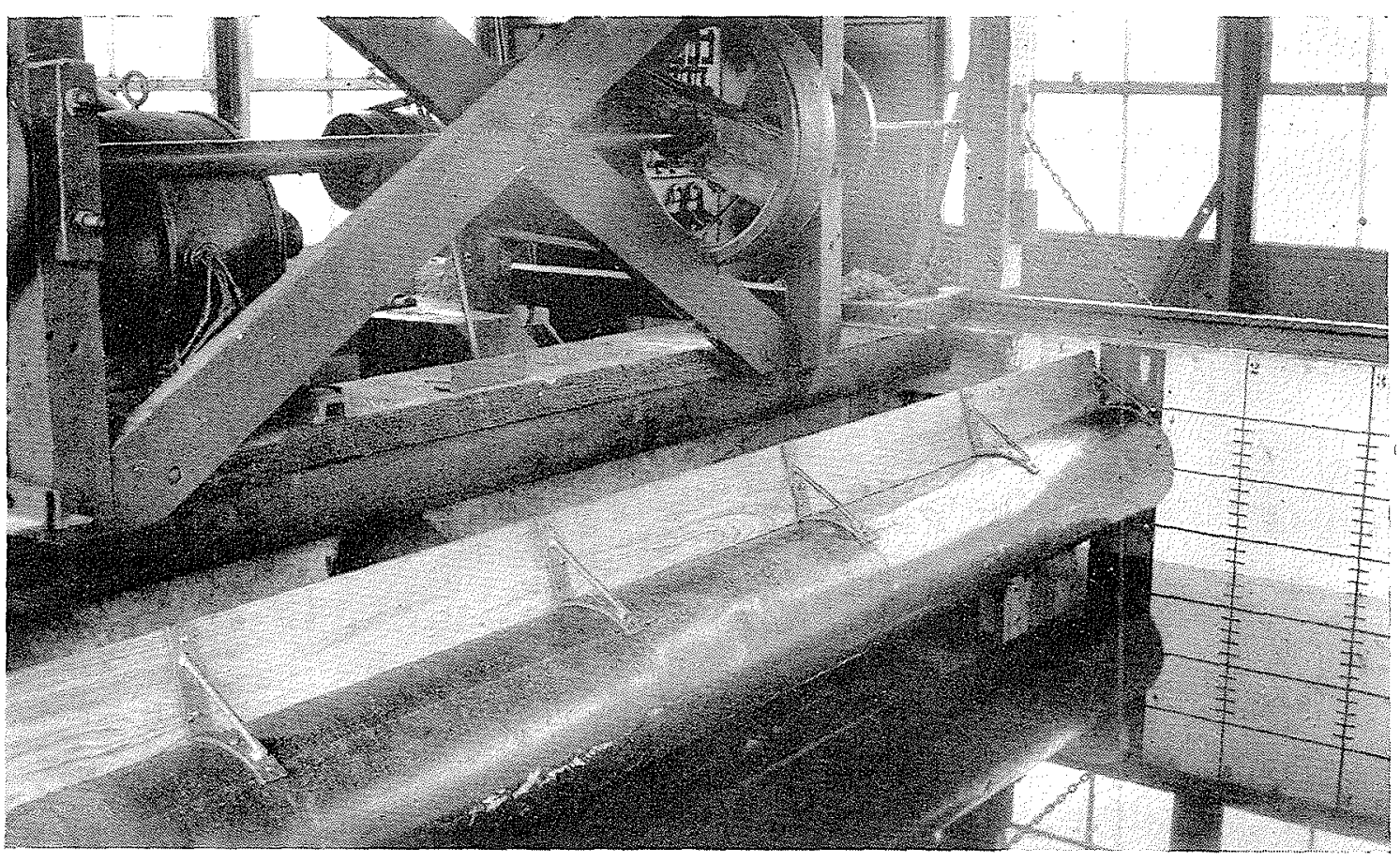

Photographie communiquée par le "Beach Erosion Board».

PRINCIPE. - Lor DU MOUVEMENT :

Cet appareil est constitué par un cylindre tournant muni d'une ou plusieurs palelles.

Le mouvement imprimé aux pariicules d'eau, assez éloigné de celui de la houle au voisinage du batteur se régularise à mesure que la distance parcollrue augmente.

\section{Calcul de l'amplitude :}

La théorie exposée dans cet article ne peut fournir lamplitude de la houle produite par cet appareil.

\section{Constructron :}

Il n'y a pas d'excentrique et de manivelle; la construction est done simple.
INEITTE :

Si l'appareil est bien équilibré, l'inertie est faible.

\section{Pouvotr RÉFLÉchISSANT :}

II est peu important, puisque la palette productrice de houle n'est immergée que pendant une fraction de la période.

\section{Remarque :}

Cet appareil peut être indiqué dans certains cas particuliers, les modèles à fonds mobiles, par cxemple. 


\section{FICHE N*15}

\section{Roue à palettes}
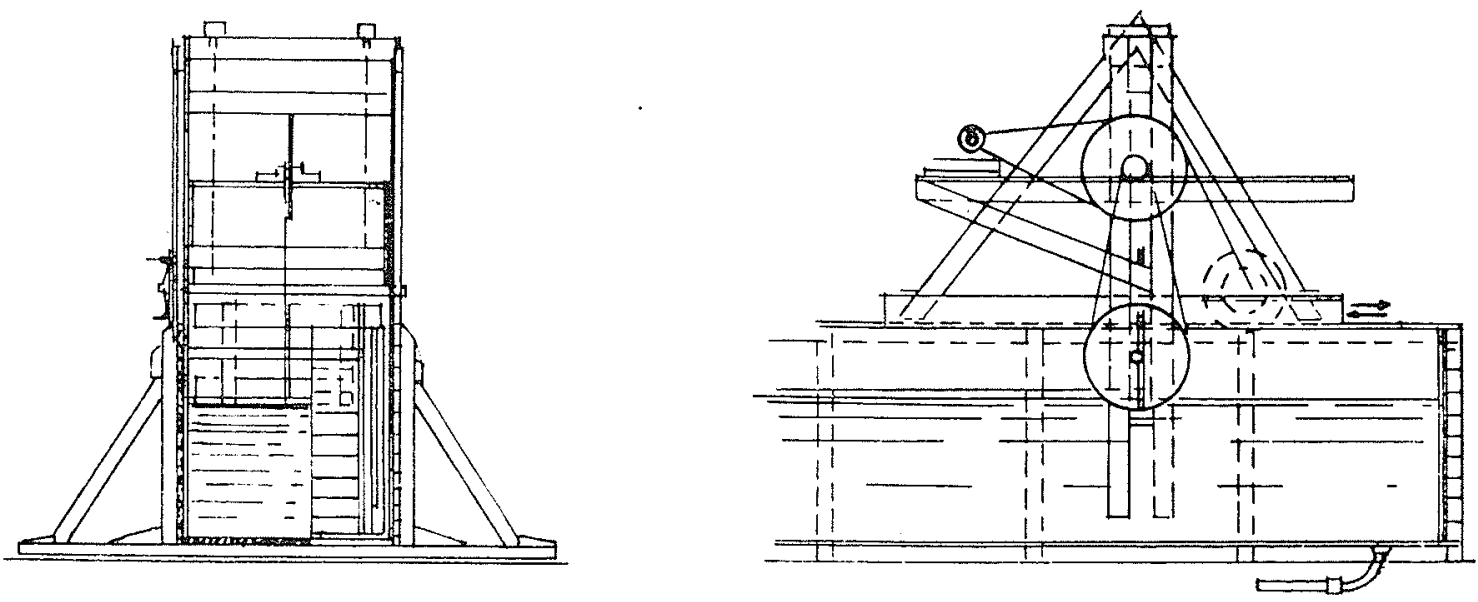

Extrait de Hydralic Laboratory Practice, 1926-1929.

Prixche. - LoI De MOUVEMENT:

Une roue munie de palettes tourne au-dessus du canal, de facon à ce que ses palettes battent leau.

Le mouvement imprimé aux particules d'eau, assez éloigné de celui de la houle au voisinage du batteur, se régularise à mesure que la distance parcourue augmente.

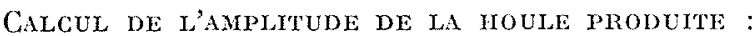

La théorie exposée dans cet article ne peut donner l'amplitude de la houle produite.

\section{INERTME :}

Le batteur est très satisfaisant à ce point de vute, ćtant donné que le corps tournant est écuilibré.

\section{GiNTETIEN :}

Il n'y a aucune articulation on palier sous l'cau : l'entreticn est done minime.

\section{RÉGLAgE :}

L'amplitude peut se régler dans une certaine mesure en élevant ou abaissant l'ensemble de l'ap. pareil.

\section{POUVOIR RÉFLÉCHISSANT :}

L'appareil est très satisfaisant de ce point de vue: il est très peu réfléchissant puisque les palettes ne sont immergees dans l'eau que pendant une fraction de la période.

\section{Remarque :}

Cet appareil peut être indiqué dans certains cas particuliers, les modèles à fond mobile par exemple. 


\section{FICHE $N^{0} 16$}

\section{Appareil pneumatique}

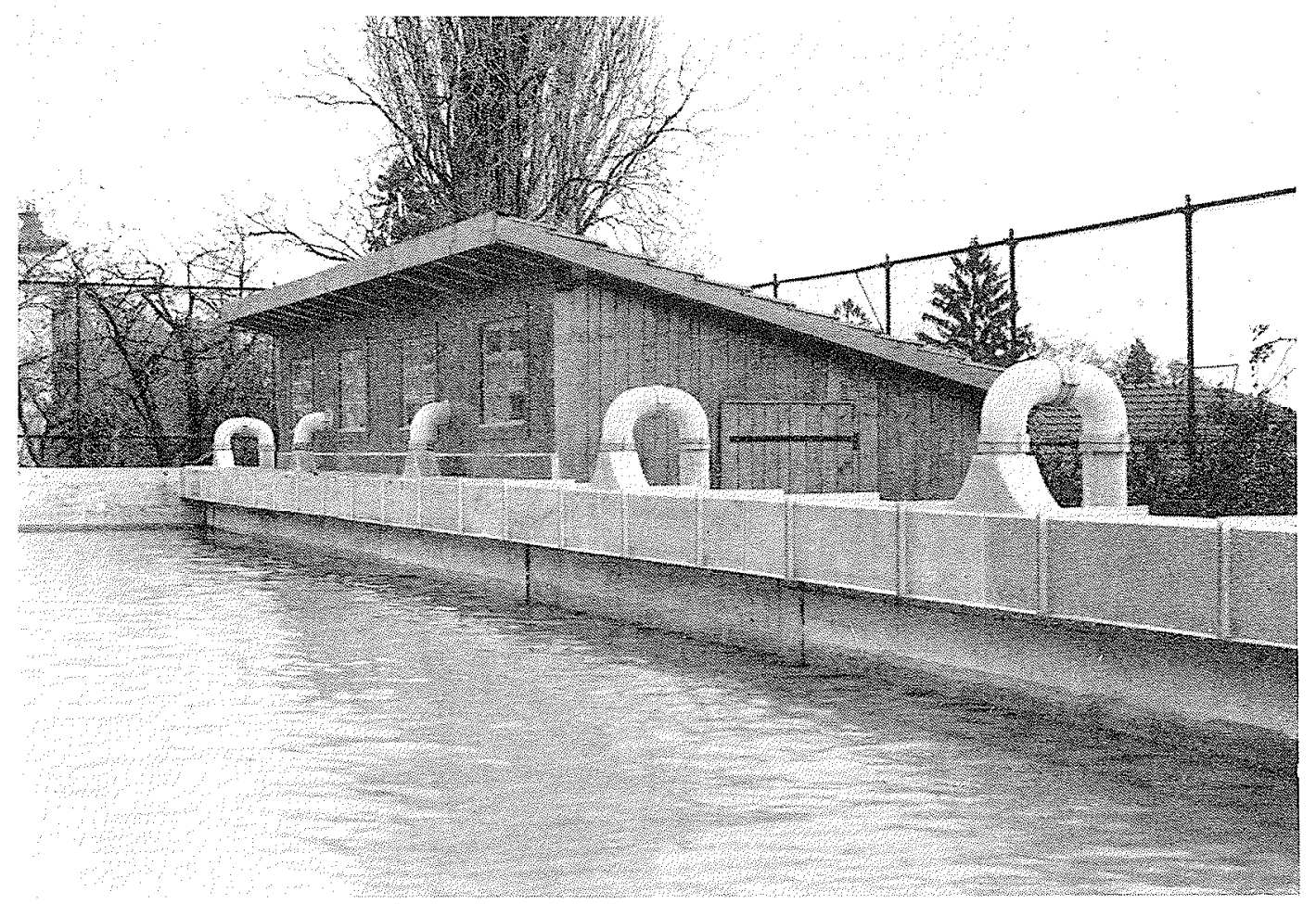

Photographic communiquée par le Laboratoire d'Hydraulique de l'Université de Lausanne.

Vue de la « eloche $\gg$ du générateur pneumatique, le bassin étant en cours de remplissage. Longueur 10tale de la « cloche $\gg: 23 \mathrm{~m}$.

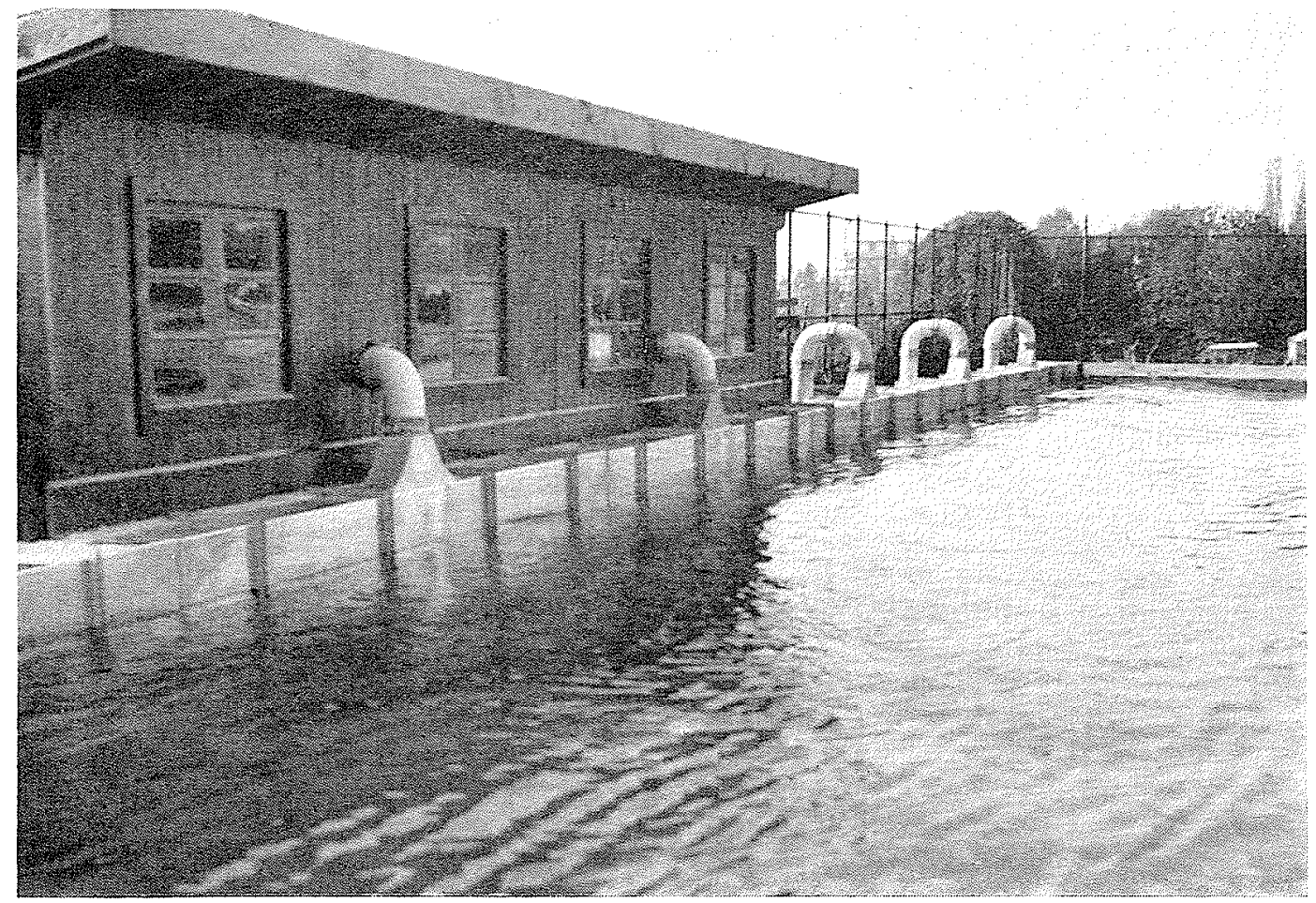

Photographie communiquée par le laboratoire dHydrauligue de l'Université de Lansanne.

Vue de la « eloche $\gg$ du générateur pneumatique, le bassin étant plein. A l'intérieur de la maison se trouvent les deux pompes à air actionnées par un moteur a explosion; la course ef la vitesse des pompes alternatives sont réglables à volonté. 


\section{FICHE N*16 (SUITE)}
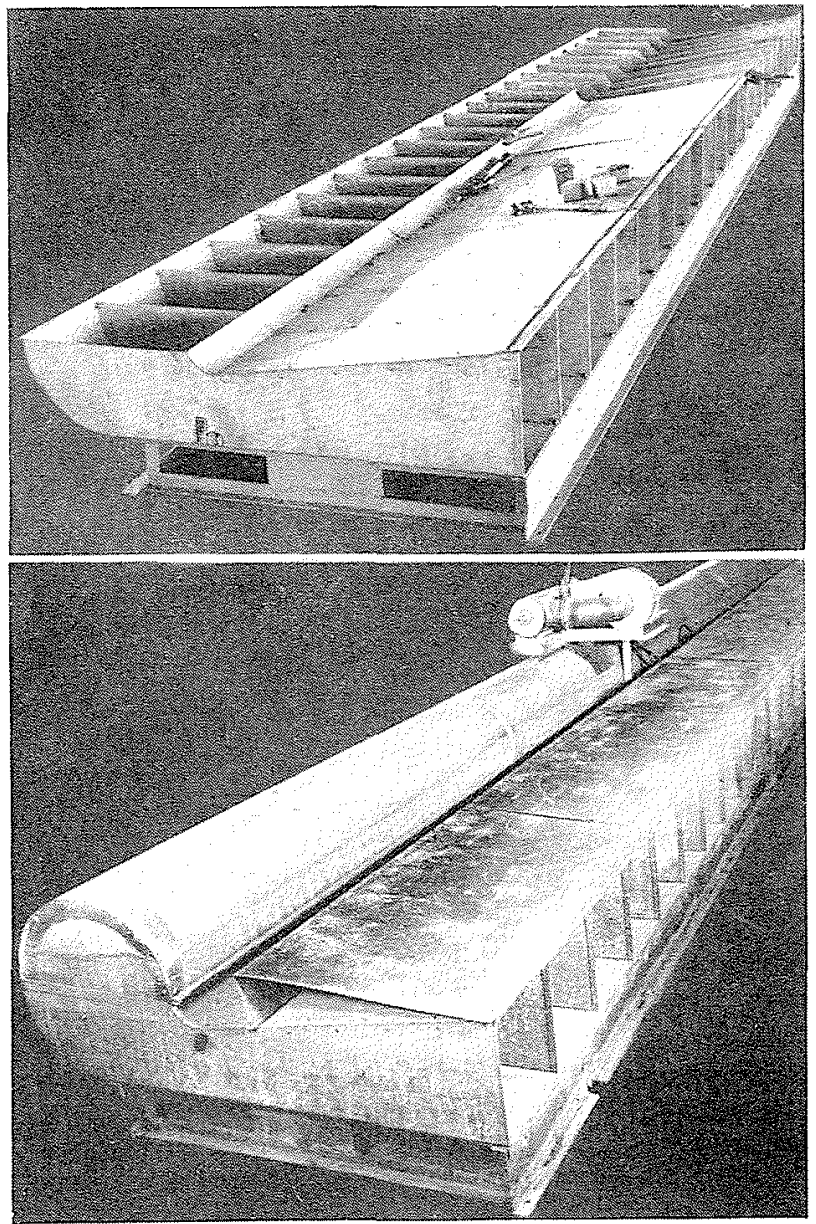

Pholographies extraites du rapport: "Model studies of Apra Harbor Guam $\bowtie$. California Institate of Technology.

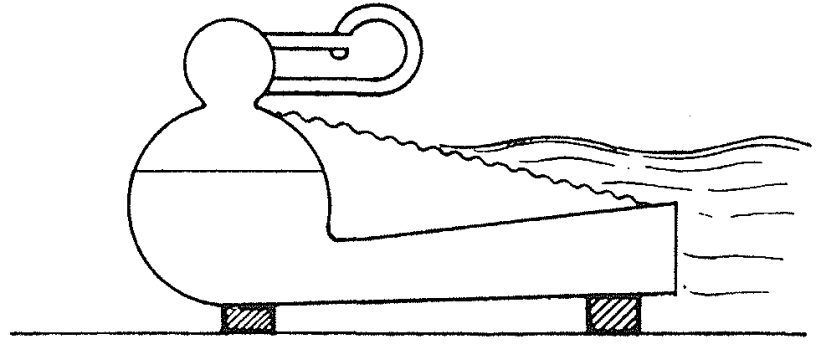

\section{Principe. - JoI de MoUvemant :}

Le principe consiste à aspirer un certain rolume d'eau dans une chambre puis à le refouler; en étudiant la forme de la chambre, on arrive à produire une houle pure $\dot{a}$ une distance relativement faible de l'appareil.

C.AICUL DE L'AMPLTTUDE DE LA HOULE PRODUITE :

La théorie exposée dans cet article ne peut évidemment donner la valeur de l'amplitude obtenue.

\section{Construction. - Entretien :}

La construction est très simple; l'appareil est robuste puisqu'il n'existe aucune pièce ou organe en mouvement dans l'eau.

\section{Moßilití: :}

Il est également très léger, cette qualité jointe à sa simplicité fait qu'il peut être monté de façon à être orienté facilement. 